INPLASY

PROTOCOL

To cite: $X u$ et al. Clinical efficacy and safety of Xingpi Yanger granule combined with Zinc gluconate in treating infantile enteritis:A systematic review and meta-analysis. Inplasy protocol 2020110128. doi:

10.37766/inplasy2020.11.0128

Received: 28 November 2020

Published: 28 November 2020

Corresponding author:

Xilian Zhang

zxI2072@126.com

Author Affiliation:

First Teaching Hospital Of

Tianjin University Of

Traditional Chinese Medicine

Support: No Funding.

Review Stage at time of this submission: Data analysis.

Conflicts of interest: No conflicts of interest.

\section{Clinical efficacy and safety of Xingpi Yanger granule combined with Zinc gluconate in treating infantile enteritis: A systematic review and meta-analysis}

Xu, J1; Zhang, X²; Fu, Q3; Zhang, Z4; Song, Y5; Zhao, T6; Tian, Y7.

Review question / Objective: Types of participants£rall infant patients diagnosed with enteritis (aged 5 and under, with no lower age limit) will be included in the study. We will follow the Medicine clinical practice guidelines of acute infectious diarrhea from 2018 Children" As Hospital of Zhejiang University School or Diagnosis and Guidelines for clinical Practice of Acute infectious diarrhoeal diseases in Children in China from 2016 Chinese Journal of Pediatrics. Types of interventions£rboth the experimental group and the control group routinely treated for enteritis diseases, including rehydration,diet treatment and other non-drug treatment. According to the literature, the intervention methods were designed into Xingpiyanger granule combined with Zinc gluconate. Types of comparison£rpatients in the control group were designed into antibiotic combined with montmorillonite powder. Types of outcomes£rilf the temperature of the child drops gradually and the vomiting symptoms are relieved within 72 hours of the treatment, it will be considered effective.

INPLASY registration number: This protocol was registered with the International Platform of Registered Systematic Review and Meta-Analysis Protocols (INPLASY) on 28 November 2020 and was last updated on 28 November 2020 (registration number INPLASY2020110128).

\section{INTRODUCTION}

Review question / Objective: Types of participants£̧िAll infant patients diagnosed with enteritis (aged 5 and under, with no lower age limit) will be included in the study. We will follow the Medicine clinical practice guidelines of acute infectious diarrhea from 2018 Children" AEs Hospital of Zhejiang University School or Diagnosis and Guidelines for clinical Practice of Acute infectious diarrhoeal diseases in 
Children in China from 2016 Chinese Journal of Pediatrics. Types of interventions - Both the experimental group and the control group routinely treated for enteritis diseases, including rehydration, diet treatment and other non-drug treatment. According to the literature, the intervention methods were designed into Xingpiyanger granule combined with Zinc gluconate. Types of comparison: Patients in the control group were designed into antibiotic combined with montmorillonite powder. Types of outcomes: If the temperature of the child drops gradually and the vomiting symptoms are relieved within $\mathbf{7 2}$ hours of the treatment, it will be considered effective.

Condition being studied: Infantile diarrheal disease is a common clinical disease. When children suffer from acute diarrhea, infectious gastroenteritis is the most common cause, which is often accompanied by symptoms such as vomiting and fever, and dehydration and poisoning can occur in moderate and severe cases.If not timely treatment will have a serious impact on the growth and development of children or life safety. At present, the main therapies for infantile enteritis include rehydration, zinc supplementation, diet control, probiotics and other means. Those with high fever and severe poisoning symptoms can be treated with antibiotics, but the cycle of therapy is long, and the problems such as antibiotic abuse and bacterial resistance still exist. Xingpi Yanger granule, a Chinese patent medicine, often used in the treatment of pediatric digestive system diseases such as indigestion, diarrhea.It has very strong adsorption capacity for bacteria, viruses and toxins, solid bowel and anti-diarrhea effect, greatly enhancing the function of intestinal mucosal surface adsorption, avoiding intestinal mucosa damage caused by the pathogen microorganism. Therefore, the clinical commonly used auxiliary treatment of infantile diarrhea. The traditional Chinese medicine theory suggests the granule also work for the diarrhea caused by pathogenic factors, impairment by overeating and Spleen-Stomach Weakness.

\section{METHODS}

Search strategy: In this study, we will search 8 commonly used databases:Cochrane Library, PubMed, Web of Science, EMBASE, the Chinese Biology Medicine (CBM), the China Science and Technology Journal Database (VIP), ØCthe China National Knowledge Infrastructure Database (CNKI), and WanFang database. The retrieval time begins on the database" As build date and ends in September 2020. The references of included studies will be tracked and those meeting the inclusion criteria will be supplemented. The search strategy will be adjusted according to the characteristics of different databases.

Participant or population: All infant patients with no other complications diagnosed with enteritis (aged 5 and under, with no lower age limit) will be included in the study. We will follow the Medicine clinical practice guidelines of acute infectious diarrhea from 2018 Children" Es Hospital of Zhejiang University School or Diagnosis and Guidelines for clinical Practice of Acute infectious diarrhoeal diseases in Children in China from 2016 Chinese Journal of Pediatrics.

Intervention: Both the experimental group and the control group routinely treated for enteritis diseases, including rehydration, diet treatment and other non-drug treatment. According to the literature, the intervention methods were designed into Xingpiyanger granule combined with Zinc gluconate.

Comparator: Patients in the control group were designed into antibiotic combined with montmorillonite powder.

Study designs to be included: All available randomized controlled trials (RCTs) that investigated the efficacy and safety of treatment of Xingpiyanger granule combined with Zinc gluconate in patients diagnosed with enteritis will be included in this systematic review.Articles without sufficient available data, noncomparative studies, non-RCTs, literature reviews, 
meta-analysis, meeting abstracts, and case reports will be excluded.

Eligibility criteria: (1)Types of trials: Randomized controlled trials (RCTs) on Xingpiyanger granule combined with Zinc gluconate for treating enteritis were included.(2) Types of patients:All infant patients diagnosed with enteritis (aged 5 and under, with no lower age limit) will be included in the study, following the Medicine clinical practice guidelines of acute infectious diarrhea from 2018 Children" Es Hospital of Zhejiang University School or Diagnosis and Guidelines for clinical Practice of Acute infectious diarrhoeal diseases in Children in China from 2016 Chinese Journal of Pediatrics. (3)Types of interventional measures:the control group was treated with antibiotic combined with montmorillonite powder, and other routine treatments, while the experimental group was administered Xingpiyanger granule combined with Zinc gluconate and routine therapeutic interventions. (4) Types of outcome measures: the primary outcome indicator was total clinical efficacy [total clinical efficacy $=$ (number of obvious cases + number of effective cases)/(total number of cases)"'[100\%]. Effective cases were defined as remission of the fever and vomiting in $72 \mathrm{~h}$.

Information sources: In this study, we will search 8 commonly used databases: Cochrane Library, PubMed, Web of Science, EMBASE, the Chinese Biology Medicine (CBM), the China Science and Technology Journal Database (VIP), ØCthe China National Knowledge Infrastructure Database (CNKI), and WanFang database.

\section{Main outcome(s): 1) Effective. 2) Adverse} event.

Additional outcome(s): $\phi \nvdash t h e$ fever time; фŚduration of vomiting; $₫ U \overline{d e h y d r a t i o n ~ r e l i e f ~}$ time; $₫ U ̈ d i a r r h e a$ duration; $₫ Z ̇ h o s p i t a l i z a t i o n$

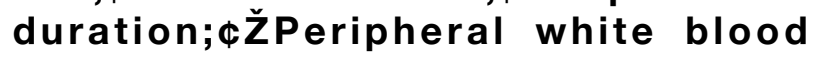
cell(WBC) recovery; $\$ B S t o o l s$ white blood cell(S-WBC) recovery.
Data management: The authors independently extracted the data from the included studies by a standardised Excel form. We collected the following data:author, year, sample size, age, the dosage of XPYE and zinc gluconate and the dosage of antibiotics and montmorillonite powder. We checked the data for erratum, retraction, fraud, and inconsistencies.

Quality assessment / Risk of bias analysis: Two reviewers independently evaluated the risk of bias for the included studies using the Cochrane Collaboration" Es tool for evaluating the risk of bias, consensus, or consulting with the third author if necessary. This tool supports the consideration of sequence generation, allocation concealment, blinding, incomplete outcome data, selective outcome reporting, and other sources of bias. We assessed the risk of bias in each source as low risk of bias, high risk of bias, and unclear risk of bias and contacted the study" IEs authors to request for missing information by using open-ended questions if necessary.

Strategy of data synthesis: RevMan 5.3 was used for the meta-analysis of multiple studies.We will detect treatment effect of dichotomous data as risk ratio and $95 \%$ confidence intervals, and will estimate treatment effect of continuous data as mean difference or standardized mean difference and $95 \%$ confidence intervals.

Subgroup analysis: A subgroup analysis will be conducted to explore the major heterogeneity based on the course of treatment.

Sensibility analysis: A sensitivity analysis will be performed to examine the satiability of study findings by eliminating low quality studies.

Language: There are Chinese and English in search.

Country(ies) involved: All of the authors and institutions are from China. 
Other relevant information: Xingpi Yanger granule; Zinc gluconate; Infantile enteritis; efficacy; safety.

Keywords: Xingpi Yanger granule; Infantile enteritis; efficacy; safety; Meta-analysis.

Contributions of each author:

Author 1 - Jiabao Xu.

Email: 1766837232@qq.com

Author 2 - Xilian Zhang.

Email: zx12072@126.com

Author 3 - Qianfang Fu.

Email: 1728020603@qq.com

Author 4 - Zhenhua Zhang.

Email: zzh28920@163.com

Author 5 - Ying Song.

Email: 18730155703@163.com

Author 6 - Ting Zhao.

Email: 1042293850@qq.com

Author 7 - Yuling Tian.

Email: 2325108492@qq.com 